\title{
BIFURCATION OF LIMIT CYCLES: GEOMETRIC THEORY
}

\author{
L. M. PERKO
}

(Communicated by Kenneth R. Meyer)

\begin{abstract}
Multiple limit cycles play a basic role in the theory of bifurcations. In this paper we distinguish between singular and nonsingular, multiple limit cycles of a system defined by a one-parameter family of planar vector fields. It is shown that the only possible bifurcation at a nonsingular, multiple limit cycle is a saddle-node bifurcation and that locally the resulting stable and unstable limit cycles expand and contract monotonically as the parameter varies in a certain sense. Furthermore, this same type of geometrical behavior occurs in any oneparameter family of limit cycles experiencing a saddle-node type bifurcation except possibly at a finite number of points on the multiple limit cycle.
\end{abstract}

\section{INTRODUCTION}

This paper contains some basic new information concerning bifurcations at multiple limit cycles of a planar system

$$
\dot{x}=f(x, \lambda)
$$

depending on a parameter $\lambda \in \mathbf{R}$. It is assumed that $f$ is an analytic function; although, in discussing bifurcations at a limit cycle of multiplicty $m$, it suffices to assume that $f$ is of class $C^{m}$.

We distinguish between two types of multiple limit cycles, singular and nonsingular multiple limit cycles, and prove that the bifurcation theory for nonsingular, multiple limit cycles is exactly the same as the bifurcation theory for multiple limit cycles belonging to a one-parameter family of vector fields; cf. $[5,9]$. In other words, the only possible type of bifurcation that can occur at a nonsingular, multiple limit cycle $\Gamma_{0}$ of $\left(1_{\lambda}\right)$ is a saddle-node bifurcation in which $\Gamma_{0}$ bifurcates into two hyperbolic limit cycles, one stable and the other unstable, which expand and contract monotonically as the parameter $\lambda$ varies in a certain sense (described in Table 1 in $\S 2$ ). We also prove that this type of geometrical behavior occurs in any one-parameter family of limit cycles that experiences a saddle-node type of bifurcation except possibly at a finite number of points on the multiple limit cycle.

The proofs, as well as the definition of a singular, multiple limit cycle, are based on certain properties of the Poincare map; they utilize the Weierstrass

Received by the editors March 25, 1990.

1980 Mathematics Subject Classification (1985 Revision). Primary 58F14, 58F21.

This work was supported by a Northern Arizona University Organized Research Grant. 
preparation theorem and some of the same techniques, based on Puiseux series, that were employed by the author in [8].

\section{BifURCATION AT NONSINGULAR MULTIPLE LIMIT CYCLES}

Assume that the system $\left(1_{\lambda}\right)$ has a limit cycle

$$
\Gamma_{0}: x=\gamma_{0}(t)
$$

at some parameter value, say $\lambda=0$, and let $T_{0}$ denote the minimum period of the periodic function $\gamma_{0}(t)$. For convenience in notation, we define the function $f_{0}(t)=f\left(\gamma_{0}(t), 0\right)$, and let $\nabla \cdot f_{0}(t)$ denote the function $\nabla \cdot f\left(\gamma_{0}(t), 0\right)$. We also define $\omega_{0}$ to be \pm 1 according to whether $\Gamma_{0}$ is positively or negatively oriented respectively. For planar systems, we utilize the following classical definition of a multiple limit cycle; cf. [1, p. 118].

Definition 1. The limit cycle $\Gamma_{0}$ is a multiple limit cycle of $\left(1_{0}\right)$ if

$$
\int_{0}^{T_{0}} \nabla \cdot f_{0}(t) d t=0 .
$$

In order to understand the meaning of this definition and, indeed, to understand the theory of bifurcation of limit cycles, one must be familiar with the Poincare map. This idea originated with Poincaré [10] at the turn of the century. In order to define the Poincare map, let $l_{\tau}$ be the straight line normal to the limit cycle $\Gamma_{0}$ at the point $\gamma_{0}(\tau) \in \Gamma_{0}$ and let $s$ denote the signed distance along $l_{\tau}$ with $s>0$ on the exterior of $\Gamma_{0}$ and $s<0$ on the interior of $\Gamma_{0}$. The existence and analyticity of the Poincaré map $h(s, \lambda, \tau)$ for $|s|<\delta,|\lambda|<\delta$, $\tau \in \mathbf{R}$, and some $\delta>0$ is established in [1,7]. Cf. [8, Lemma 1.1 and Remark 1.1]. In terms of the Poincaré map, $h(s, \lambda, \tau)$, we define the displacement function

$$
d(s, \lambda, \tau)=h(s, \lambda, \tau)-s .
$$

See Figure 1. If the point $\gamma_{0}(\tau)$ on $\Gamma_{0}$ plays no role in a particular use of the displacement function $d(s, \lambda, \tau)$, then we simply denote the displacement function by $d(s, \lambda)$.

The following result is classical (cf. [1, p. 383]).

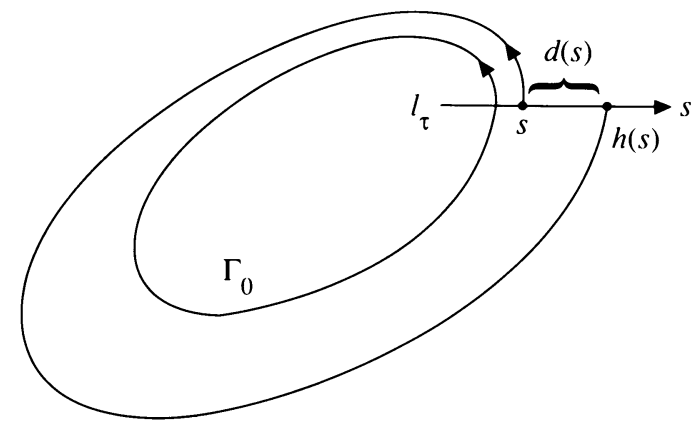

Figure 1. (The Poincaré map and displacement function for $\left(1_{\lambda}\right)$ in a neighborhood of the limit cycle $\Gamma_{0}$ of $\left(1_{0}\right)$.) 
Lemma 1. For $\delta>0,|s|<\delta$, and $|\lambda|<\delta$, let $d(s, \lambda)$ denote the displacement function for the system $\left(1_{\lambda}\right)$. Then the derivative $d_{s}(0,0)$ is independent of the point $\gamma_{0}(\tau)$ on $\Gamma_{0}$ and

$$
d_{S}(0,0)=e^{\int_{0}^{T_{0}} \nabla \cdot f_{0}(t) d t}-1 .
$$

We see that $\Gamma_{0}$ is a 8multiple limit cycle of $\left(1_{0}\right)$ if and only if for all $\tau \in \mathbf{R}$, that $d_{s}(0,0, \tau)=0$; and this is equivalent to saying that $\Gamma_{0}$ has a characteristic multiplier equal to one (cf. [7]).

Definition 2. If

$$
d(0,0)=d_{s}(0,0)=d_{s}^{(2)}(0,0)=\cdots=d_{s}^{(m-1)}(0,0)=0
$$

and

$$
d_{s}^{(m)}(0,0) \neq 0
$$

then, for $m>1, \Gamma_{0}$ is called a multiple limit cycle of multiplicity $m$. If $m=1$, i.e., if $d(0,0)=0$ and $d_{s}(0,0) \neq 0$, then $\Gamma_{0}$ is called a simple limit cycle or hyperbolic limit cycle.

Remark 1. If follows from Theorem $42[1$, p. 277] that the multiplicity $m$ of a multiple limit cycle $\Gamma_{0}$ is independent of the point $\gamma_{0}(\tau)$ on $\Gamma_{0}$; in fact, according to Theorem 42 in [1], the multiplicity $m$ of $\Gamma_{0}$ is equal to the maximum number of limit cycles that can bifurcate from $\Gamma_{0}$ under a perturbation of $\left(1_{0}\right)$. And for analytic systems,

$$
d(0,0)=d_{s}(0,0)=d_{s}^{(2)}(0,0)=\cdots=0
$$

if and only if $\Gamma_{0}$ is a cycle that belongs to a continuous band of cycles (cf. [1] or [7]).

As was pointed out by Chicone and Jacobs [3], one of the most important results for planar systems $\left(1_{\lambda}\right)$, depending on a parameter $\lambda \in \mathbf{R}$, is the following result, which is established in [1, p. 384]. (The wedge product of two vectors $x=\left(x_{1}, x_{2}\right)$ and $y=\left(y_{1}, y_{2}\right)$ is defined by $\left.x \wedge y=x_{1} y_{2}-y_{1} x_{2}\right)$.

Lemma 2. For $\delta>0,|s|<\delta,|\lambda|<\delta$, and $\tau \in \mathbf{R}$, let $d(s, \lambda, \tau)$ denote the displacement function for the system $\left(1_{\lambda}\right)$ along the normal line $l_{\tau}$ and let $\omega_{0}$ denote the orientation of $\Gamma_{0}$. Then

$$
d_{\lambda}(0,0,0)=-\frac{\omega_{0} e^{\int_{0}^{T_{0}} \nabla \cdot f_{0}(t) d t}}{\left|f_{0}(0)\right|} \int_{0}^{T_{0}} e^{-\int_{0}^{t} \nabla \cdot f_{0}(u) d u} f \wedge f_{\lambda}\left(\gamma_{0}(t), 0\right) d t .
$$

First we extend the result in Lemma 2 and determine the dependence of $d(0,0, \tau)$ on $\tau$, i.e., on the point $\gamma_{0}(\tau) \in \Gamma_{0} \cap l_{\tau}$.

Lemma 3. Under the hypotheses of Lemma 2,

$$
\begin{aligned}
d_{\lambda}(0,0, \tau) & =-\frac{\omega_{0} e^{\int_{\tau}^{\tau+T_{0}} \nabla \cdot f_{0}(t) d t}}{\left|f_{0}(\tau)\right|} \int_{\tau}^{\tau+T_{0}} e^{-\int_{\tau}^{t} \nabla \cdot f_{0}(u) d u} f \wedge f_{\lambda}\left(\gamma_{0}(t), 0\right) d t . \\
& =-\frac{\omega_{0} e^{\tau+T_{0}} \nabla \cdot f_{0}(t) d t}{\left|f_{0}(\tau)\right|} \int_{\tau}^{\tau+T_{0}} e^{-\int_{0}^{t} \nabla \cdot f_{0}(u) d u} f \wedge f_{\lambda}\left(\gamma_{0}(t), 0\right) d t .
\end{aligned}
$$

This lemma is proved by replacing the functions $\gamma_{0}(t)$ and $f_{0}(t)$ in equation (3) by the functions $\gamma_{0}(t+\tau)$ and $f_{0}(t+\tau)$ respectively and then showing that the result reduces to equation (4). 
Remark 2. The integral containing the wedge product in equation (4) is related to the Melnikov function, which plays such an important role in the theory of perturbed dynamical systems (cf. [6 or 7]). We define the function

$$
M(\tau)=\int_{\tau}^{\tau+T_{0}} e^{-\int_{\tau}^{t} \nabla \cdot f_{0}(u) d u} f \wedge f_{\lambda}\left(\gamma_{0}(t), 0\right) d t
$$

for later use.

Our first new result, which forms the basis for much of this paper, is the following:

Theorem 1. Under the hypotheses of Lemma 2, if $\Gamma_{0}$ is a multiple limit cycle of $\left(1_{0}\right)$, then

$$
d_{\lambda}(0,0, \tau)=\frac{\left|f_{0}(0)\right|}{\left|f_{0}(\tau)\right|} e^{\int_{0}^{\tau} \nabla \cdot f_{0}(t) d t} d_{\lambda}(0,0,0)
$$

Proof. By definition, $\Gamma_{0}$ is a multiple limit cycle if and only if

$$
\int_{0}^{T_{0}} \nabla \cdot f_{0}(t) d t=0 .
$$

Thus, since $\nabla \cdot f_{0}(t)$ is a $T_{0}$-periodic function of mean value zero, it follows that

$$
\int_{0}^{t+T_{0}} \nabla \cdot f_{0}(u) d u=\int_{0}^{t} \nabla \cdot f_{0}(u) d u .
$$

And then, since $f \wedge f_{\lambda}\left(\gamma_{0}(t), 0\right)$ is a $T_{0}$-periodic function of $t$, it follows that the integrand in expression (4) in Lemma 3 is a $T_{0}$-periodic function of $t$. Therefore, it follows from Lemmas 2 and 3 that

$$
\begin{aligned}
d_{\lambda}(0,0, \tau) & =-\frac{\omega_{0} e^{\int_{0}^{\tau+T_{0}} \nabla \cdot f_{0}(t) d t}}{\left|f_{0}(\tau)\right|} \int_{\tau}^{\tau+T_{0}} e^{-\int_{0}^{t} \nabla \cdot f_{0}(u) d u} f \wedge f_{\lambda}\left(\gamma_{0}(t), 0\right) d t \\
& =-\frac{\omega_{0} e^{\int_{0}^{\tau} \nabla \cdot f_{0}(t) d t}}{\left|f_{0}(\tau)\right|} \int_{0}^{T_{0}} e^{-\int_{0}^{t} \nabla \cdot f_{0}(u) d u} f \wedge f_{\lambda}\left(\gamma_{0}(t), 0\right) d t \\
& =\frac{\left|f_{0}(0)\right|}{\left|f_{0}(\tau)\right|} e^{\int_{0}^{\tau} \nabla \cdot f_{0}(t) d t} d_{\lambda}(0,0,0) .
\end{aligned}
$$

Corollary 1. Under the hypotheses of Theorem $1, d_{\lambda}(0,0,0)$ is positive, zero, or negative if and only if for all $\tau \in \mathbf{R}, d_{\lambda}(0,0, \tau)$ is positive, zero, or negative respectively.

Definition 3. The limit cycle $\Gamma_{0}$ is a singular, multiple limit cycle of $\left(1_{0}\right)$ if

$$
d_{s}(0,0)=0 \text { and } d_{\lambda}(0,0)=0 .
$$

If $d_{s}(0,0)=0$ and $d_{\lambda}(0,0) \neq 0$, then $\Gamma_{0}$ is a nonsingular, multiple limit cycle of $\left(1_{0}\right)$.

Remark 3. Note that $\Gamma_{0}$ is a singular, multiple limit cycle of $\left(1_{0}\right)$ if and only if

$$
\int_{0}^{T_{0}} \nabla \cdot f_{0}(t) d t=0
$$

and

$$
\int_{0}^{T_{0}} e^{-\int_{0}^{t} \nabla \cdot f_{0}(u) d u} f \wedge f_{\lambda}\left(\gamma_{0}(t), 0\right) d t=0
$$


Definition 4. $\mu(\tau)=\operatorname{sgn} M(\tau)=\operatorname{sgn} \int_{\tau}^{\tau+T_{0}} e^{-\int_{0}^{t} \nabla \cdot f_{0}(u) d u} f \wedge f_{\lambda}\left(\gamma_{0}(t), 0\right) d t$.

Corollary 2. Under the hypotheses of Theorem 1 , if $\Gamma_{0}$ is a nonsingular, multiple limit cycle of $\left(1_{0}\right)$, then for all $\tau \in \mathbf{R}, \mu(\tau)=\mu(0)$.

Since, by definition, any one-parameter family of rotated vector fields $f(x, \lambda)$ satisfies

$$
f \wedge f_{\lambda}(x, \lambda)>0
$$

(cf. [5] or [9]). We immediately obtain the following:

Theorem 2. If $f(x, \lambda)$ defines a one-parameter family of rotated vector fields in $\mathbf{R}^{2}$ with parameter $\lambda \in \mathbf{R}$, then any multiple limit cycle of $\left(1_{\lambda}\right)$ is a nonsingular, multiple limit cycle of $\left(1_{\lambda}\right)$.

In order to present our main result for nonsingular, multiple limit cycles, we define $\sigma_{0}$ to be \pm 1 according to whether the limit cycle $\Gamma_{0}$ is unstable or stable on its exterior respectively; and we define $\mu_{0}=\mu(0)$ where $\mu(\tau)$ is defined in Definition 4. Then if $\Gamma_{0}$ is a nonsingular, multiple limit cycle of $\left(1_{0}\right)$, it follows from Corollary 2 that for all $\tau \in \mathbf{R}, \mu(\tau)=\mu_{0}$.

Our main result for nonsingular, multiple limit cycles is a simple application of the implicit function theorem (since $d_{\lambda}(0,0) \neq 0$ ) (cf. [8]). The following result generalizes the results for one-parameter families of rotated vector fields contained in Theorem 8 in [5], the results contained in Theorem F in [9], and the results contained in Theorems 71,72 in [1]. It shows that the behavior at a nonsingular, multiple limit cycle of $\left(1_{\lambda}\right)$ is exactly the same as the behavior at a multiple limit cycle in a one-parameter family of rotated vector fields; i.e., the only bifurcation that can occur at a nonsingular, multiple limit cycle $\Gamma_{0}$ is a saddle-node bifurcation where $\Gamma_{0}$ splits into two simple limit cycles of the opposite stability.

Theorem 3. If $\Gamma_{0}$ is a nonsingular, multiple limit cycle of $\left(1_{0}\right)$, then $\Gamma_{0}$ belongs to a unique, one-parameter family of limit cycles of $\left(1_{\lambda}\right)$ and

(1) if the multiplicity of $\Gamma_{0}$ is odd, then the family either expands or contracts monotonically as $\lambda$ increases through zero as determined by Table 1 while

(2) if the multiplicity of $\Gamma_{0}$ is even, then $\Gamma_{0}$ bifurcates into a simple stable limit cycle and a simple unstable limit cycle as $\lambda$ varies in a certain sense, determined by Table 1, and $\Gamma_{0}$ disappears as $\lambda$ varies in the opposite sense.

Remark 4. The stable and unstable limit cycles generated in a saddle-node bifurcation at a nonsingular, multiple limit cycle $\Gamma_{0}$ of even multiplicity, $m$, also

\begin{tabular}{|c|c|c|c|c|}
\hline$\omega_{0}$ & + & + & - & - \\
\hline$\sigma_{0}$ & + & - & + & - \\
\hline$\mu_{0} \Delta \lambda$ & + & - & - & + \\
\hline
\end{tabular}

Table 1. The change in $\lambda, \Delta \lambda$, that causes the expansion of a nonsingular, multiple limit cycle $\Gamma_{0}$ of odd multiplicity or the bifurcation of a nonsingular, multiple limit cycle $\Gamma_{0}$ of even multiplicity. 


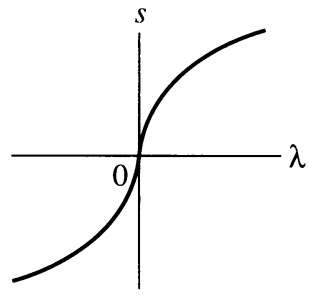

(a) m odd

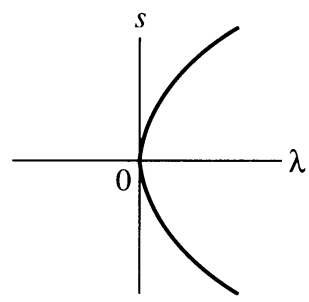

(b) m even

FIGURE 2. The curve $d(s, \lambda)=0$ describing a oneparameter family of limit cycles of $\left(1_{\lambda}\right)$.

expand or contract monotonically for sufficiently small $\lambda$ as described by Table 1. For example, if $\Gamma_{0}$ is a positively oriented, nonsingular, multiple limit cycle that is unstable on its exterior (i.e., $\omega_{0}=+1$ and $\sigma_{0}=+1$ ), then for $\mu_{0}=+1$, it follows from Table 1 that $\Gamma_{0}$ bifurcates into a simple stable limit cycle $\Gamma_{\lambda}^{-}$ and a simple unstable limit cycle $\Gamma_{\lambda}^{+}$as the parameter $\lambda$ increases. Furthermore, for sufficiently small $\lambda>0, \Gamma_{\lambda}^{-} \subset \operatorname{Int} \Gamma_{0}, \Gamma_{\lambda}^{+} \subset \operatorname{Ext} \Gamma_{0}$ and in this case $\Gamma_{\lambda}^{+}$expands monotonically and $\Gamma_{\lambda}^{-}$contracts monotonically away from $\Gamma_{0}$ as $\lambda$ increases from zero. The bifurcation diagram for this case is shown in Figure 2(b). When $\Gamma_{0}$ is a nonsingular, multiple limit cycle, the curve $d(s, \lambda)=0$ is described by a function $\lambda=\lambda(s)=s^{m}\left(b_{0}+b_{1} s+b_{2} s^{2}+\cdots\right)$ for $|s|<\delta$ and some $\delta>0$ according to the implicit function theorem. Although the coefficients $b_{i}=b_{i}(\tau)$ depend on the point $\gamma_{0}(\tau) \in \Gamma_{0} \cap l_{\tau}, b_{0}(\tau) \neq 0$ for $\tau \in \mathbf{R}$ since $d_{\lambda}(0,0, \tau) \neq 0$ for $\tau \in \mathbf{R}$; in fact, $\operatorname{sgn}\left[b_{0}(\tau)\right]=\omega_{0} \sigma_{0} \mu_{0}$ for all $\tau \in \mathbf{R}$. In the case when $\omega_{0} \sigma_{0} \mu_{0}=+1$, the nature of the curve $d(s, \lambda)=0$ for $m$ odd or even is shown in Figure $2(\mathrm{a})$ or $2(\mathrm{~b})$ respectively. In case $\omega_{0} \sigma_{0} \mu_{0}=-1$, the curve $d(s, \lambda)=0$ can be obtained by rotating the curves shown in Figure 2 about the $s$-axis.

If $\Gamma_{0}$ is a simple limit cycle, then at any point $\gamma_{0}(\tau)$ on $\Gamma_{0}, d_{s}(0,0, \tau) \neq 0$ and it follows from the implicit function theorem that for each $\tau \in \mathbf{R}$ the curve $d(s, \lambda, \tau)=0$ is described by a function $s=s(\lambda, \tau)$ and the rate of growth of the limit cycle $\Gamma_{0}$ with resepct to $\lambda$ at the point $\gamma_{0}(\tau) \in \Gamma_{0}$ is determined by

$$
\frac{\partial s}{\partial \lambda}(0, \tau)=-\frac{d_{\lambda}(0,0, \tau)}{d_{s}(0,0, \tau)}
$$

(cf. [5, equation (3.17)]). It follows from Lemmas 1 and 3 that

$$
\operatorname{sgn}\left[\frac{\partial s}{\partial \lambda}(0, \tau)\right]=\omega_{0} \sigma_{0} \mu(\tau),
$$

since a simple limit cycle is stable (or unstable) if and only if

$$
\int_{0}^{T_{0}} \nabla \cdot f_{0}(t) d t<0 \quad(\text { or }>0),
$$

i.e., $\operatorname{sgn}\left[d_{s}(0,0)\right]=\sigma_{0}$ (cf. $[1$, p. 118$]$ or $[7$, Chapter $\left.3, \S 4]\right)$. These results can be summarized as follows:

Theorem 4. If $\Gamma_{0}$ is a simple limit cycle of $\left(1_{0}\right)$, then $\Gamma_{0}$ belongs to a unique, one-parameter family of limit cycles $\Gamma_{\lambda}$ of $\left(1_{\lambda}\right)$ and at any point $\gamma_{0}(\tau)$ on $\Gamma_{0}$, 
increasing the parameter $\lambda$ causes the limit cycle $\Gamma_{\lambda}$ to expand or contract along the normal line $l_{\tau}$ if and only if $\omega_{0} \sigma_{0} \mu(\tau)= \pm 1$ respectively.

An example of a one-parameter family of limit cycles $\Gamma_{\lambda}$ that expands along various portions of $\Gamma_{0}$ and contracts along other portions of $\Gamma_{0}$ is furnished by the last example in $\S 2$ of [8]. Of course, if $\mu(\tau)$ is of one sign, as in the case when $f(x, \lambda)$ defines a one-parameter family of rotated vector fields, then according to Theorem 4 , the simple limit cycle $\Gamma_{0}$ expands or contracts monotonically as $\lambda$ increases through zero as determined by Table 1 . This generalizes the results for families of rotated vector fields contained in Theorem 7 in [5] and Theorem $\mathrm{D}$ in [9].

\section{BifURCATION AT SINGULAR MULTiPLE Limit CyCles}

In $\S 2$ we saw that if $\Gamma_{0}$ is a simple limit cycle or a nonsingular, multiple limit cycle of $\left(1_{0}\right)$, then there is only one branch of $d(s, \lambda)=0$ passing through the origin and the only bifurcation that can occur at $\Gamma_{0}$ is a saddle-node bifurcation. On the other hand, if $\Gamma_{0}$ is a singular, multiple limit cycle of multiplicity $m$, then it follows from the Weierstrass preparation theorem that there may be as many as $m$ branches of $d(s, \lambda)=0$ passing through the origin (cf. [8]).

If $\Gamma_{0}$ is a singular, multiple limit cycle of $\left(1_{0}\right)$ that belongs to a oneparameter family of limit cycles $\Gamma_{\lambda}$, then as in [8], $\Gamma_{\lambda}$ is defined by a branch $s(\lambda, \tau)$ of $d(s, \lambda, \tau)=0$, which can be expanded in a Puiseux series

$$
s(\lambda, \tau)=(\sigma \lambda)^{k / m} \sum_{i=0}^{\infty} a_{i}(\tau)(\sigma \lambda)^{i / m}
$$

for $0 \leq \sigma \lambda<\sigma \delta$ where $\sigma$ is $\pm 1, a_{0}(\tau) \neq 0$ except possibly at finitely niany $\tau \in\left[0, T_{0}\right), \delta$ is some positive constant and $k$ and $m$ are unique relatively prime positive integers (cf. [1] or [4]). In this case $\Gamma_{0}$ is said to be a multiple limit cycle belonging to a one-parameter family of limit cycles $\Gamma_{\lambda}$ of reduced multiplicity $m$. For simplicity in notation, we consider the point $\tau \in\left[0, T_{0}\right)$ as fixed in what follows and delete the dependence of the displacement function on $\tau$ even though the coefficeints $a_{i}(\tau)$ in the Puiseux series (5) can depend on $\tau$; cf. Remark 5 below.

In order to illustrate the type of geometrical information that can be obtained from the Puiseux series (5), consider the case when $\sigma=+1$ and $a_{0}>0$. For $m$ odd, let $\beta=\lambda^{1 / m}$. Then there is a unique analytic continuation of the function

$$
s\left(\beta^{m}\right)=\beta^{k} \sum_{i=0}^{\infty} a_{i} \beta^{i},
$$

defined by (5) for $0 \leq \beta<\delta$, to an interval $|\beta|<\delta$. And for $m$ even, $k$ is odd, and the inverse of the function $s(\lambda)$ exists and satisfies

$$
\lambda(s)=s^{m / k} \sum_{i=0}^{\infty} b_{i} s^{i / k}
$$

for $0 \leq s<\delta$, where $b_{0}>0$ and $\delta$ is some positive constant. Then since $k$ is odd, just as above, this function can be unqiuely continued to a function defined by (6) on an interval $|s|<\delta$. Now let $\lambda=\beta^{m}$ and $\beta= \pm \lambda^{1 / m}$ with 


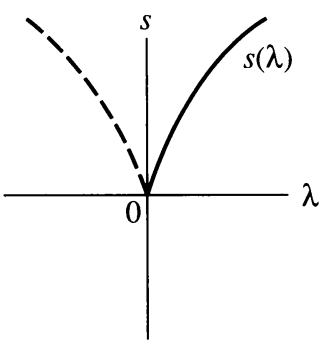

(a) m odd

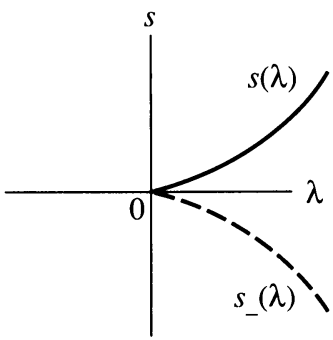

(b) m even

FIGURE 3. Typical branches of $d(s, \lambda)=0$ along a fixed normal line $l_{\tau}$ to the limit cycle $\Gamma_{0}$.

$\operatorname{sgn}(\beta)=\operatorname{sgn}(s)$. Then from $(6)$,

$$
\beta=s^{1 / k}\left[\sum_{i=0}^{\infty} b_{i} s^{i / k}\right]^{1 / m}
$$

for $|s|<\delta$. Since $k$ is odd, this function is one-to-one for $|s|<\delta$ and its inverse is the unique analytic continuation of the function $s\left(\beta^{m}\right)$, defined by the Puiseux series (5) for $0 \leq \beta<\delta$, to an interval $|\beta|<\delta$. This is equivalent to continuing the upper branch of $s(\lambda)$ given by (5) to the lower branch given by

$$
s_{-}(\lambda)=-\lambda^{k / m} \sum_{i=0}^{\infty}(-1)^{i} a_{i} \lambda^{i / m}
$$

for $0 \leq \lambda<\delta$; and this continuation is unique. Note that for $\sigma=+1$, the functions $s(\lambda), \lambda(s)$ and $s_{-}(\lambda)$, defined by (5), (6), and (7) respectively, satisfy $s(\lambda(x))=x, s_{-}(\lambda(-x))=-x$ and $\lambda(s(x))=\lambda\left(s_{-}(x)\right)=x$ for all $x$ in $[0, \delta)$. A typical bifurcation diagram for the branch $s(\lambda)$ of $d(s, \lambda)=0$ and its continuation $s_{-}(\lambda)$ when $m$ is even is shown in Figure 3(b). A typical branch $s(\lambda)$ of $d(s, \lambda)=0$ and its continuation when $m$ is odd is shown in Figure 3(a).

Similar results follow when $\sigma$ or $a_{0}$ is negative and this leads to the following result:

Theorem 5. Suppose that $\Gamma_{0}$ is a singular, multiple limit cycle of $\left(1_{0}\right)$ that belongs to a one-parameter family of limit cycles $\Gamma_{\lambda}$ of $\left(1_{\lambda}\right)$, corresponding to a branch $s(\lambda, \tau)$ of $d(s, \lambda, \tau)=0$, of reduced multiplicity $m$. Then for all but possibly a finite number of $\tau \in\left[0, T_{0}\right)$, there is a $\delta>0$ such that $s(\lambda, \tau)$ can be expanded in a Puiseux series, (5) with $a_{0}(\tau) \neq 0$, which converges for $0 \leq \sigma \lambda<\sigma \delta$ where $\sigma= \pm 1$; furthermore,

(1) if $m$ is even, then $\Gamma_{0}$ bifurcates into a simple stable limit cycle and a simple unstable limit cycle belonging to the family $\Gamma_{\lambda}$ as $\sigma \lambda$ increases and $\Gamma_{0}$ disappears as $\sigma \lambda$ decreases;

(2) if $m$ is odd and $k$ is odd, then the limit cycles of the family $\Gamma_{\lambda}$ expand or contract along the normal line $l_{\tau}$ to $\Gamma_{0}$ as $\sigma \lambda$ increases according to whether $a_{0}(\tau)$ is positive or negative respectively; and

(3) if $m$ is odd and $k$ is even, then the limit cycles of the family $\Gamma_{\lambda}$ expand or contract along the normal line $l_{\tau}$ to $\Gamma_{0}$ as $\lambda$ increases in $(0, \delta)$ or 
as $\lambda$ decreases in $(-\delta, 0)$ according to whether $a_{0}(\tau)$ is positive or negative respectively.

Corollary 3. Under the hypotheses of Theorem 5 , if the reduced multiplicity $m$ is even, the leading coefficient $a_{0}(\tau)$ in the Puiseux series (5) does not change sign.

This corollary follows immediately from Theorem 5 since for $m$ even, the simple unstable and stable limit cycles $\Gamma_{\lambda}^{ \pm}$in part (1) of Theorem 5 correspond to the branches $s_{ \pm}(\lambda, \tau)$ or to the branches $s_{\mp}(\lambda, \tau)$ of $d(s, \lambda, \tau)=0$ where $s_{ \pm}(\lambda, \tau)$ are defined by $(5)$ and (7) respectively. But then if $a_{0}(\tau)$ changes sign, this would imply that for all sufficiently small $\sigma \lambda>0$ the limit cycles $\Gamma_{\lambda}^{+}$and $\Gamma_{\lambda}^{-}$of $\left(1_{\lambda}\right)$ intersect, a contradiction. Thus, $a_{0}(\tau)$ does not change sign.

Remark 5. The relatively prime integers $m$ and $k$ in the Puiseux series (5) are unique and independent of the point $\gamma_{0}(\tau) \in \Gamma_{0}$ through which the normal line $l_{\tau}$ passes. In fact, all possible values of $\mathrm{k} / \mathrm{m}$ are given by the absolute values of the slopes of the sides of the Newton polygon for the displacement function $d(s, \lambda, \tau)$ as described in [1] or [4]. However, the coefficients $a_{0}(\tau)$ can depend on $\tau$ and for $m$ odd, $a_{0}(\tau)$ can change sign as $\tau$ varies in $\left[0, T_{0}\right)$. Thus, Theorem 5 tells us whether at particular point $\gamma_{0}(\tau) \in \Gamma_{0}$, the family of limit cycles $\Gamma_{\lambda}$ moves inward or outward along the normal line $l_{\tau}$ as the parameter $\lambda$ increases through zero. It does not imply that the family $\Gamma_{\lambda}$ expands or contracts monotonically in a neighborhood of $\Gamma_{0}$ unless $a_{0}(\tau)$ is of a constant sign. Since the coefficient $a_{0}(\tau)$ is either very difficult or impossible to determine in terms of the vector field $f(x, \lambda)$ and the periodic orbit $\gamma_{0}(t)$, Theorem 5 does not appear to be of much practical value; however, it is the basis for the theorem establishing that any one-parameter family of limit cycles can be continued through a bifurcation in a unique way, proved in [8], as well as for the next geometrical theorem.

Theorem 6. Under the hypotheses of Theorem 5 , if $m$ is even, it follows that $\Gamma_{0}$ bifurcates into a simple stable limit cycle $\Gamma_{\lambda}^{-}$and a simple unstable limit cycle $\Gamma_{\lambda}^{+}$as the parameter $\lambda$ varies in one sense and that $\Gamma_{0}$ disappears as $\lambda$ varies in the opposite sense. Furthermore, except at a finite number of points on $\Gamma_{0}$, either $\Gamma_{\lambda}^{+}$expands monotically away from $\Gamma_{0}$ and $\Gamma_{\lambda}^{-}$contracts monotonically away from $\Gamma_{0}$ as the parameter $\lambda$ varies monotonically in a neighborhood of zero or vica versa.

Proof. If the reduced multiplicity $m$ in equation (5) is even, then at any point $\gamma_{0}(\tau) \in \Gamma_{0} \cap l_{\tau}$ where $a_{0}(\tau) \neq 0$ the graph of the continuation of the inverse function $\lambda(s)$, given by equation (6), for $|s|<\delta$ has the form of one of the functions (of $s$ ) shown in Figure 4 (or one of these graphs rotated about the $s$-axis).

According to Theorem 5, for $m$ even, $\Gamma_{0}$ bifurcates into a simple stable limit cycle $\Gamma_{\lambda}^{-}$and a simple unstable limit cycle $\Gamma_{\lambda}^{+}$as $\sigma \lambda$ increases. And, according to Corollary $3, a_{0}(\tau)$ does not change sign. Thus, since $a_{0}(\tau)$ is analytic, $a_{0}(\tau)$ is either positive or negative except possibly at a finite number of points in $\left[0, T_{0}\right]$. Assume that $a_{0}(\tau)>0$ except possibly at a finite number of points in $\left[0, T_{0}\right]$ and that the simple unstable and stable limit cycles $\Gamma_{\lambda}^{ \pm}$in part (1) of Theorem 5 correspond to the branches $s_{ \pm}(\lambda, \tau)$ of $d(s, \lambda, \tau)=0$ 

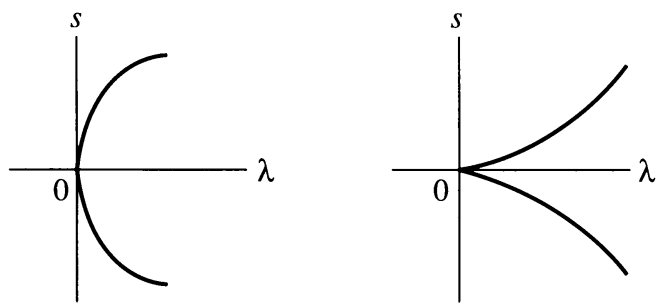

FIGURE 4. The graph of the function $\lambda(s)$ for $|s|<\delta$.

defined by (5) and (7) respectively. It then follows from the nature of the graphs of the functions $s_{ \pm}(\lambda)$, shown in Figure 4 (for $\sigma=+1$ ), that at any point $\gamma_{0}(\tau) \in \Gamma_{0} \cap l_{\tau}$, except possibly at the points $\gamma_{0}\left(\tau^{*}\right)$ where $a_{0}\left(\tau^{*}\right)=0, \Gamma_{\lambda}^{+}$ expands monotonically away from $\Gamma_{0}$ and $\Gamma_{\lambda}^{-}$contracts monotonically away from $\Gamma_{0}$ as $\sigma \lambda$ increases monotonically from zero. The other cases, where $a_{0}(\tau)<0$ except possibly at a finite number of points in $\left[0, T_{0}\right]$ or where $\Gamma_{\lambda}^{ \pm}$ correspond to the branches $s_{\mp}(\lambda, \tau)$ respectively, can be treated similarly, and therefore, we have the conclusion of Theorem 6 .

Remark 6 . Theorem 6 implies that at every point $\gamma_{0}(\tau)$ on $\Gamma_{0}$, except possibly at a finite number of points $\gamma_{0}\left(\tau^{*}\right)$ where $a_{0}\left(\tau^{*}\right)=0$, the simple unstable and stable limit cycles $\Gamma_{\lambda}^{ \pm}$expand and contract monotonically away from $\Gamma_{0}$ as $\sigma \lambda$ increases from zero. On the other hand, both $\Gamma_{\lambda}^{+}$and $\Gamma_{\lambda}^{-}$may expand (or contract) monotonically away from $\Gamma_{0}$ in a neighborhood of a point $\gamma_{0}\left(\tau^{*}\right)$ where $a_{0}\left(\tau^{*}\right)=0$ as shown in Figure 5 .

For example, suppose that for $0 \leq \lambda<\delta$

$$
s_{+}(\lambda, \tau)=\lambda^{3 / 2}\left[a_{0}(\tau)+a_{1}(\tau) \lambda^{1 / 2}+\cdots\right]
$$

Then, according to equation (7),

$$
s_{-}(\lambda, \tau)=-\lambda^{3 / 2}\left[a_{0}(\tau)-a_{1}(\tau) \lambda^{1 / 2}+\cdots\right]
$$

for $0 \leq \lambda<\delta$ and if $a_{0}\left(\tau^{*}\right)=0$, it follows that

$$
s_{ \pm}\left(\lambda, \tau^{*}\right)=\lambda^{2}\left[a_{1}\left(\tau^{*}\right) \pm a_{2}\left(\tau^{*}\right) \lambda^{1 / 2}+\cdots\right]
$$

for $0 \leq \lambda<\delta$. The nature of the functions $s_{ \pm}\left(\lambda, \tau^{*}\right)$ is then shown in Figure

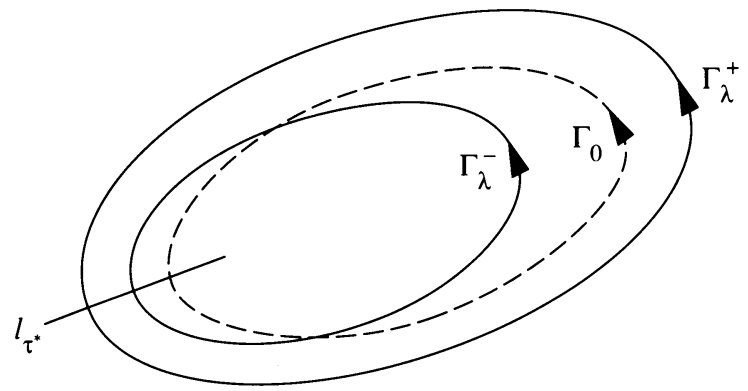

FIgURE 5. An example of a limit cycle $\Gamma_{\lambda}^{-}$, which neither exapnds nor contracts monotonically away from $\Gamma_{0}$ with a variation of the parameter $\lambda$. 


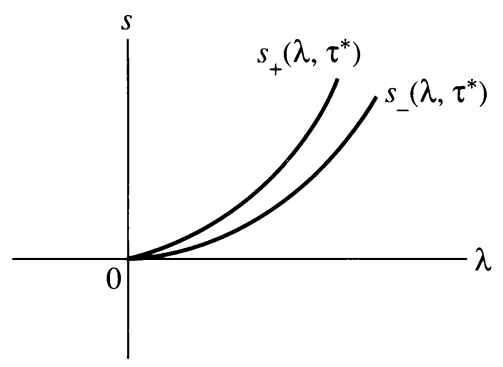

Figure 6. The graphs of the branches $s_{ \pm}\left(\lambda, \tau^{*}\right)$ of $d\left(s, \lambda, \tau^{*}\right)=0$ at a point $\gamma_{0}\left(\tau^{*}\right) \in \Gamma_{0} \cap l_{\tau^{*}}$ where $a_{0}\left(\tau^{*}\right)$ $=0$.

6 (for $a_{1}\left(\tau^{*}\right)>0$ and $\left.a_{2}\left(\tau^{*}\right)>0\right)$ and the behavior of the limit cycles $\Gamma_{\lambda}^{ \pm}$near

the point $\gamma_{0}\left(\tau^{*}\right) \in \Gamma_{0} \cap l_{\tau^{*}}$ is shown in Figure 5 in this case.

The type of behavior described in Remark 6 and Figure 5 apparently occurs near the points $\gamma_{0}\left( \pm \tau^{*}\right)$ where the $y$-axis intersects the semistable limit cycle $\Gamma_{0}$, which occurs at the bifurcation value $\lambda_{0}=6.557853 \ldots$, of the system

$$
\dot{x}=-y+\lambda x-(1+\lambda) x^{3} / 3+x^{5} / 5 \quad \dot{y}=x .
$$

(A study of this sytem was suggested by the referee.) This system is symmetric with respect to the origin and a numerical study of this system shows that the unstable limit cycle $\Gamma_{\lambda}^{+}$expands monotonically away from $\Gamma_{0}$ at all points on $\Gamma_{0}$ and that the stable limit cycle $\Gamma_{\lambda}^{-}$contracts monotonically away from $\Gamma_{0}$ except in a neighborhood of the points $\gamma_{0}\left( \pm \tau^{*}\right)$ where, as $\lambda$ increases from $\lambda_{0}$, $\Gamma_{\lambda}^{-}$expands monotonically away from $\Gamma_{0}$ (as in Figure 5). It is conjectured that $a_{0}\left( \pm \tau^{*}\right)=0$ where $\gamma_{0}\left( \pm \tau^{*}\right)$ are the points where $\Gamma_{0}$ intersects the $y$-axis in this example.

\section{Conclusions}

To summarize, any bifurcation at a periodic orbit of a planar analytic system $\left(1_{\lambda}\right)$ depending on a parameter $\lambda \in \mathbf{R}$ occurs at a multiple limit cycle of $\left(1_{\lambda}\right)$ or at a cycle belonging to a continuous band of cycles of $\left(1_{\lambda}\right)$. The only bifurcation that occurs at a nonsingular, multiple limit cycle is the saddle-node bifurcation with the stable and unstable bifurcating limit cycles expanding and contracting monotonically. On the other hand, as many as $m$ one-parameter families of limit cycles can bifurcate from a singular, multiple limit cycle of multiplicity $m$; however, any bifurcating one-parameter family whose reduced multiplicity is even corresponds to a saddle-node type of bifurcation that exhibits the same geometrical behavior as in the nonsingular case except possibly at a finite number of points on the multiple limit cycle. It is conjectured that this same type of geometrical behavior characterizes the saddle-node type of bifurcation in any number of dimensions.

It was shown by the author in [8] that at most a finite number of oneparameter families of limit cycles can bifurcate from any cycle belonging to a continuous band of cycles and it can be shown, using the Weierstrass preparation theorem and Puiseux series, exactly as in this paper, that even in this case any bifurcating one-parameter family of even reduced multplicity corresponds 
to a saddle-node type of bifurcation whose limit cycles exhibit the same kind of monotone expansion and contraction as described in Theorem 6. Several interesting examples of one-parameter families of limit cycles bifurcating from a center have recently been studied by Chicone and Jacobs [3] and Blows and Perko [2].

\section{REFERENCES}

1. A. A. Andronov et al., Theory of bifurcations of dynamical systems on a plane, Kefer Press, Jerusalem, 1971.

2. T. R. Blows and L. M. Perko, Bifurcation of limit cycles from centers, S.I.A.M. J. Math. Anal.submitted.

3. C. Chicone and M. Jacobs, Bifurcation of limit cycles from quadratic isochrones, J. Differential Equations 91 (1991), 268-326.

4. S. N. Chow and J. K. Hale, Methods of bifurcation theory, Springer-Verlag, New York, 1982.

5. G. F. D. Duff, Limit cycles and rotated vector fields, Ann. of Math. (2) 67 (1953), 15-31.

6. J. Guckenheimer and P. Holmes, Nonlinear oscillations, dynamical systems and bifurcations of vector fields, Appl. Math. Sci., vol. 42, Springer-Verlag, New York, 1983.

7. L. M. Perko, Differential equations and dynamical systems, Texts in Appl. Math., vol. 7, Springer-Verlag, New York, 1990.

8. __ Global families of limit cycles of planar analytic systems, Trans. Amer. Math. Soc., 322 (1990), 627-656.

9. __ Rotated vector fields and the global behavior of limit cycles for a class of quadratic systems in the plane, J. Differential Equations 18 (1975), 63-86.

10. H. Poincaré, Mémorie sur les courbes définies par une equation différentielle, J. Mathématiques 7 (1881), 375-422; Oeuvre, Gauthier-Villar, Paris, 1880-1890, pp. 1-221.

Department of Mathematics, Northern Arizona University, Flagstaff, Arizona 86011 